Check for updates

Cite this: J. Mater. Chem. B, 2017, 5, 9165

Received 6th July 2017.

Accepted 8th November 2017

DOI: $10.1039 / c 7 t b 01825 h$

rsc.li/materials-b

\section{Electrospun organic-inorganic nanohybrids as sustained release drug delivery systems $\dagger$}

\author{
Yanshan Gao, ${ }^{\mathrm{ab}}$ Tian Wei Teoh, ${ }^{\mathrm{b}}$ Qiang Wang (D)*a and Gareth R. Williams (D) *b
}

\begin{abstract}
In this work, the non-steroidal anti-inflammatory drugs ibuprofen (ibu) and ketoprofen (ket), both poorly soluble in water, were first intercalated into layered double hydroxide (LDH) nanoparticles. The drug$\mathrm{LDH}$ composites were then mixed with poly( $\varepsilon$-caprolactone) $(\mathrm{PCL})$ at $5 \%$ and $10 \% \mathrm{w} / \mathrm{w}$ ratios and processed into fibers via electrospinning, yielding organic-inorganic nanohybrids. PCL/drug fibers were additionally prepared as controls. The average diameter of the fibers ranged from 400 to $1000 \mathrm{~nm}$. The fibers are found to be smooth and cylindrical, with the $\mathrm{LDH}$-containing systems having more homogenous fibers than those without the inorganic filler. From in vitro drug release tests, it was determined that more than $90 \%$ of the intercalated ibu and ket were released from the drug-LDH nanohybrids within the first 4 hours. Similarly, more than $60 \%$ of the incorporated drug was freed from the $\mathrm{PCL} /$ drug fibers in this time period. However, the release rates of both ibu and ket from the drug-LDH loaded PCL fibers were significantly slower. Only $44-48 \%$ of ibu was released from the PCL/ibu-LDH system after 5 days, while the amount released in the case of ket was 20-25\%. In addition, drug release was still ongoing after 5 days for all the PCL/drug-LDH samples. These systems are thus proposed to have potential as implantable drug delivery systems.
\end{abstract}

\section{Introduction}

Biodegradable and biocompatible polymers have been widely explored in drug delivery. ${ }^{1}$ Amongst these, poly( $\varepsilon$-caprolactone) (PCL) has received significant attention because of its slow degradation rate ( $\approx 3$ years in vivo), which lends it to the preparation of long-lasting implants. PCL has been processed using a wide variety of pharmaceutical technologies, including with the electrospinning approach. The latter comprises a facile and scalable method to generate fibers from polymer solutions or melts. ${ }^{2,3}$

In solution electrospinning, a polymer and functional component (such as a drug) are first dissolved in a volatile solvent. The resultant solution is ejected from a syringe fitted with a metal tip towards a metal collector plate, with a high potential difference applied between the two. The electrical energy causes the solvent to evaporate rapidly, resulting in one-dimensional fibers in which the drug is typically dispersed as a solid solution in the polymer. Usually the fiber diameter ranges from a few nanometers to a few micrometers. ${ }^{4-6}$ Electrospun drug loaded fibers have been widely explored for targeted release, for improving

\footnotetext{
${ }^{a}$ College of Environmental Science and Engineering, Beijing Forestry University, 35 Qinghua East Road, Haidian District, Beijing 100083, P. R. China. E-mail: qiangwang@bjfu.edu.cn, qiang.wang.ox@gmail.com; Tel: +8613699130626

${ }^{b}$ UCL School of Pharmacy, University College London, 29-39 Brunswick Square, London WC1N 1AX, UK. E-mail: g.williams@ucl.ac.uk; Tel: +44 (0)20 77535868 $\dagger$ Electronic supplementary information (ESI) available. See DOI: 10.1039/c7tb01825h
}

the dissolution rate of poorly water-soluble drugs, and for encapsulating multiple functional components. ${ }^{7-10}$

However, when fabricating nanofibers a burst release of drug is often observed even if the polymer filament is insoluble in water (as is the case for PCL). This arises because of the very high surface area-to-volume ratio of the fibers, which results in a large proportion of the incorporated drug molecules being present at or near the fiber surfaces. These molecules can rapidly diffuse into the release medium, leading to an initial burst release. A number of authors have explored this phenomenon, ${ }^{4,11,12}$ and the principal strategy used to ameliorate burst release has been to prepare core/shell fibers with drug present in the core component only. In such systems, the drug molecules must diffuse through the blank polymer shell before exiting the fibers, and thus the burst effect can often be prevented. ${ }^{13-16}$ However, the production of core/shell fibers is not a panacea, and several studies have shown that simply having a blank polymer shell surrounding a drug-loaded core does not always prevent the initial burst of release. ${ }^{17,18}$ A potentially more powerful route to preclude burst release is using triaxial electrospinning to generate three-layer fibers with a gradient distribution of drug. ${ }^{19}$

An alternative approach is to prepare fibers loaded with drugcontaining nanoparticles. Some studies have been performed exploring this option, and it has been shown to have potential. For instance, when fibers of poly(lactic-co-glycolic acid) containing rhodamine B-loaded mesoporous silica nanoparticles were prepared, rhodamine release was slowed compared to the silica 
nanoparticles alone. ${ }^{20}$ Wang et al. ${ }^{21}$ studied composite PCL fibers containing chitosan nanoparticles loaded with naproxen or rhodamine $\mathrm{B}$, and found the properties of the drug to have a major influence on the release rate. In other work, Polacco and co-workers prepared fibers of poly(lactic acid)/\&-caprolactone copolymers containing poly(DL-lactide-co-glycolide) and gelatin nanoparticles loaded with various drugs. ${ }^{22}$ It was postulated that these fibers could have applications in targeted delivery. Despite its evident promise, however, the production of nanoparticleloaded fibers has been less explored than the core/shell fiber technique. In this work we sought to explore the use of layered double hydroxide (LDH) nanoparticles as drug carriers.

LDHs, also commonly called anionic clays, are a class of lamellar compounds made up of positively charged mixedmetal hydroxide layers with an interlayer region containing charge compensating anions and water molecules (Fig. 1(a)). They can be described by the general chemical formula $\left[\mathrm{M}_{1-x}{ }^{z+} \mathrm{M}_{x}^{3+}(\mathrm{OH})_{2}\right]^{q+}\left(\mathrm{X}^{n-}\right)_{q / n} \cdot y \mathrm{H}_{2} \mathrm{O}$, where $\mathrm{M}^{z+}$ is typically a divalent cation (such as $\mathrm{Mg}^{2+}, \mathrm{Zn}^{2+}$, or $\mathrm{Ca}^{2+}$ ) $\mathrm{M}^{3+}$ is $\mathrm{Fe}^{3+}$ or $\mathrm{Al}^{3+}$, and $\mathrm{X}$ is a charge balancing interlayer anion (which can range from simple ions such as $\mathrm{Cl}^{-}, \mathrm{NO}_{3}{ }^{-}$or $\mathrm{CO}_{3}{ }^{2-}$ to complex biomolecules). Electrostatic interactions and hydrogen bonds between the layers and the contents of the interlayer space hold adjacent layers together, forming a three-dimensional structure..$^{23,24}$ The earliest application of LDHs in relation to human health was their use as antacids and antipeptic reagents in $1996,{ }^{25}$ and in the last decade nanometer-sized LDH materials $(50-300 \mathrm{~nm})$ have been increasingly explored as drug and gene carriers and delivery systems. ${ }^{26}$ Many drugs (e.g. the non-steroidal anti-inflammatory drugs ibuprofen (ibu) and ketoprofen (ket); Fig. 1(b)) are weak acids, and thus can be deprotonated to form anions and then incorporated between the layers of an LDH system. This can lead to drug release profiles different to traditional commercial formulations. ${ }^{12,27}$

Advantages often cited for LDHs in drug delivery are that they provide protection (e.g. to UV light or heat-mediated degradation) of the incorporated anions, can improve the solubility of poorly water-soluble drugs, and can reduce side effects. ${ }^{28}$ However, like

(a)
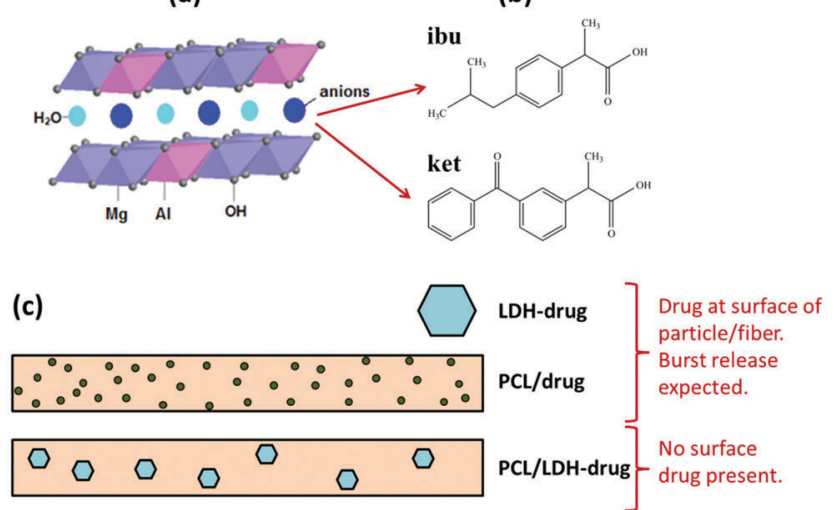

Fig. 1 A schematic diagram showing (a) the general structure of an LDH, (b) the chemical structures of ibuprofen and ketoprofen, and (c) the strategy adopted in this work. electrospun fibers LDH-drug intercalates commonly suffer from an initial burst release which cannot be controlled. In this work, we sought to incorporate drug-loaded LDH particles into PCL fibers to determine whether the production of such organic-inorganic nanohybrids can resolve this issue. A schematic diagram illustrating our approach is depicted in Fig. 1(c). Ibuprofen and ketoprofen were used as model drugs with poor solubility, and because sustained release formulations of these active ingredients could be of major benefit for the treatment of diseases such as arthritis.

To date, there are a few reports of polymer/LDH composites prepared by electrospinning in the literature, but in general such studies are focused on the preparation of advanced electronics materials or absorbents, or use the LDH to improve the mechanical properties of the fibers. For instance, Birajdar et $a .^{29}$ have used poly(vinylidene fluoride)/LDH fibers to remove $\mathrm{Cu}$ (II) ions from wastewater, and Qin and co-workers ${ }^{30}$ report that adding LDHs to poly(vinyl alcohol) fibers causes the tensile strength and elongation at break to increase. Romeo and co-workers ${ }^{31}$ generated PCL/LDH fibers using the $\mathrm{Mg} / \mathrm{Al}$ $\mathrm{LDH}$ and found that when the interlayer anion was carbonate the LDH particles were deposited at the outside of the fibers, but when the guest was 12-hydroxydodecanoate the LDH particles were largely exfoliated and evenly dispersed throughout the polymer matrix. This is expected to be because the surfactant molecules surround the hydrophilic LDH layers and reduce the interfacial tension between these and the hydrophobic PCL. The size of the LDH particles has also been shown to affect the properties of LDH-loaded electrospun fibers. ${ }^{32}$

In the biomedical setting, Shafiei et al. ${ }^{33}$ fabricated PCL composites with different LDH loadings by electrospinning and determined that the blend fibers had improved mechanical properties over pure PCL fibers, with higher tensile strength and elongation at break values. In addition, the adhesion, proliferation, and differentiation of multipotent mesenchymal stem cells were significantly improved by the addition of the LDH. There are also a few studies employing polymer/LDH fibers for drug delivery. The first report came from Tammaro and co-workers, ${ }^{2}$ who encapsulated LDH particles intercalated with diclofenac into PCL by electrospinning. PCL/diclofenac fibers exhibited an extreme burst of release, while the composite PCL/LDH-diclofenac fibers could effectively retard release and mitigate the burst.

However, this is not universally observed. Miao et al. ${ }^{11}$ produced ibuprofen drug-delivery systems from PCL and poly(lactic acid) using both the neat drug and $\mathrm{Mg} / \mathrm{Al} \mathrm{LDH}$ intercalated with ibuprofen. The use of the LDH here did not have any significant effect on the amount of burst release observed: in most cases, the release profiles leveled off very quickly (within $c a .1 \mathrm{~h}$ of the start of the release experiment), and although the presence of LDH does affect the maximum percentage release reached it does not change the overall shape of the release profiles. In other work, amoxicillin-intercalated LDH has been loaded into PCL fibers; a burst of release approaching $50 \%$ of the loading occurred during the first day of the release experiment. ${ }^{34}$ The most recent report in this area comes from Yang et al. and is 
concerned with fibers based on poly(lactic- $c o$-glycolic acid) and loaded with an $\mathrm{Mg} / \mathrm{Al} \mathrm{LDH}$ intercalated with flurbiprofen axetil. ${ }^{35}$ The presence of the LDH in the fibers could reduce the initial burst of release, and slowed down the rate at which the drug was freed into solution.

Where the polymer used for fiber formation is hydrophilic, the polar nature of the LDH particle surface should result in good compatibility between the two phases and the formation of a homogeneously-mixed composite with long term stability. In contrast, with a hydrophobic polymer such as PCL the two phases will have a tendency to separate, as noted by Romeo. ${ }^{31}$ Organic modification of the LDH surface using amphiphilic molecules such as surfactants can be used to ameliorate this problem. One route to achieve this is the aqueous miscible organic solvent treatment (AMOST), which results in stable, transparent dispersions of hydrophilic LDHs in nonpolar solvents. ${ }^{36,37}$ This simple technique uses organic solvents to wash LDH particles immediately after synthesis and can tune the surface of LDHs to be hydrophobic, enabling them to be effectively dispersed in apolar environments. ${ }^{38-40}$ AMOST modified LDHs have also been found to disperse better in hydrophobic polymers than unmodified LDH particles.

In this study, aqueous miscible organic layered double hydroxides (AMO-LDHs) were used to prepare PCL/LDH systems, with the aim of producing a homogeneous LDH dispersion in the polymer. We employed an LDH based on $\mathrm{Mg}$ and $\mathrm{Al}\left(\left[\mathrm{Mg}_{2} \mathrm{Al}(\mathrm{OH})_{6}\right] \mathrm{X} \cdot y \mathrm{H}_{2} \mathrm{O}\right.$, where $\mathrm{X}$ represents the interlayer anion) because of the biocompatibility of these metals. First, the model drugs ibuprofen and ketoprofen were intercalated into the MgAl-LDH interlayer, and the products fully characterized. Next, the LDH nanoparticles were encapsulated into a PCL matrix via electrospinning. Following an in-depth characterization of the fibers, a systematic study exploring drug release from the PCL/drug, LDH-drug and PCL/LDH-drug systems was performed.

\section{Experimental}

\subsection{Materials}

Materials were procured as follows: poly( $\varepsilon$-caprolactone) (PCL; $M_{\mathrm{n}}=70000-90000$, Sigma-Aldrich), ibuprofen sodium salt (Santa Cruz Biotechnology, $\geq 98 \%$ ), ibuprofen and ketoprofen (Sigma, $\geq 98 \%$ ), $\mathrm{MgCl}_{2} \cdot 6 \mathrm{H}_{2} \mathrm{O}$ (VWR, $99 \%$ ), $\mathrm{AlCl}_{3} \cdot 6 \mathrm{H}_{2} \mathrm{O}$ (Acros Organics, 99\%), dichloromethane (DCM; VWR, 99\%), acetone (Fisher Scientific, 99.98\%), and sodium hydroxide (Fisher Scientific).

\subsection{Synthesis of drug intercalated LDH}

Ibuprofen intercalated MgAl-LDH was synthesized using a hydrothermal method. ${ }^{40,41}$ A metal precursor solution containing $2.032 \mathrm{~g} \mathrm{MgCl}_{2} \cdot 6 \mathrm{H}_{2} \mathrm{O}$ and $1.207 \mathrm{~g} \mathrm{AlCl}_{3} \cdot 9 \mathrm{H}_{2} \mathrm{O}(\mathrm{Mg}: \mathrm{Al}=2: 1)$ in $20 \mathrm{~mL}$ deionized water was added drop-wise into a second solution comprising $2.1 \mathrm{~g}$ of sodium ibuprofen dissolved in $20 \mathrm{~mL}$ of $\mathrm{H}_{2} \mathrm{O}$. The pH was kept constant at $c a$. 9.5 using a $\mathrm{NaOH}(1 \mathrm{M})$ solution, and the subsequent slurry was transferred into a $100 \mathrm{~mL}$ Teflon-lined stainless steel autoclave.
The autoclave was sealed and a hydrothermal reaction performed for $24 \mathrm{~h}$ at $100^{\circ} \mathrm{C}$, followed by product recovery through centrifugation and washing with deionized water until the supernatant $\mathrm{pH}$ was close to 7 . A final wash was performed with acetone in order to produce AMO-LDH particles. Ketoprofen intercalated LDH was prepared in the same way, except that the $\mathrm{Mg}^{2+} / \mathrm{Al}^{3+}$ solution was added drop-wise into an alkaline solution containing $2.58 \mathrm{~g}$ of ketoprofen in $20 \mathrm{~mL} \mathrm{H}_{2} \mathrm{O}$.

\subsection{Electrospinning}

PCL solutions $(10 \% \mathrm{w} / \mathrm{v})$ were prepared by dissolving the polymer in a mixture of dichloromethane (DCM) and acetone (weight ratio $3: 1$ ) under stirring at room temperature. Ibuprofen and ketoprofen were added at concentrations of $2 \%$ and $5 \%(\mathrm{w} / \mathrm{w}$, based on the dry weight of the polymer). The solutions were then stirred again until complete dissolution of the drug had occurred. Working fluids for producing drug-LDH loaded fibers were prepared by adding the relevant nanocomposite to a $10 \% \mathrm{w} / \mathrm{v}$ PCL solution in $3: 1 \mathrm{DCM}$ : acetone, at 5 and $10 \% \mathrm{w} / \mathrm{w}$ with respect to the dry weight of the polymer.

For electrospinning, solutions were loaded into a plastic syringe fitted with a flat-tipped needle with an inner diameter of $0.61 \mathrm{~mm}$ (Nordson EFD). The solution was ejected at a speed of $0.8 \mathrm{~mL} \mathrm{~h}^{-1}$, controlled by a syringe pump (KDS100, Cole-Parmer), with a distance of $15 \mathrm{~cm}$ between the tip of the needle and the collector. A voltage of $16 \mathrm{kV}$ was applied between the needle and collector using a high-voltage power supply (HCP35-35 000, FuG Elektronik). Fibers were deposited on a flat piece of steel covered with aluminum foil, forming mats which could be peeled off for subsequent experiments.

\subsection{Characterization}

The morphologies of the LDH particles and fibers were examined using a scanning electron microscope (SEM; FEI Quanta 200 instrument) with an accelerating voltage of $5.0 \mathrm{kV}$. The samples were sputter coated with a thin gold layer before assessment to prevent charging and to improve image quality. To calculate their mean size, the diameters of the fibers were measured at more than 100 points in the SEM images. Energy dispersive X-ray spectroscopy (EDX) was carried out using a Hitachi S3400N microscope fitted with an Oxford Instruments EDX detector, and data analysed using the Inca software.

X-ray diffraction (XRD) patterns were recorded on a Rigaku MiniFlex 600 instrument in reflection mode with $\mathrm{Cu} \mathrm{K} \alpha$ radiation $(\lambda=1.5418 \AA)$. The accelerating voltage was set at $40 \mathrm{kV}$ with $15 \mathrm{~mA}$ current. Diffraction patterns were obtained over the range of $2-70^{\circ}$ at a scanning rate of $5^{\circ} \mathrm{min}^{-1}$. Fourier transform IR analyses were carried out on a Spectrum 100 instrument (PerkinElmer) over the range $650-4000 \mathrm{~cm}^{-1}$ at a resolution of $4 \mathrm{~cm}^{-1}$. Differential scanning calorimetry (DSC) analyses were performed using a Q2000 instrument (TA Instruments). Approximately $5 \mathrm{mg}$ of each sample was loaded into an aluminum pan and heated from 0 to $160{ }^{\circ} \mathrm{C}$ at a rate of $10{ }^{\circ} \mathrm{C} \mathrm{min}{ }^{-1}$ under a nitrogen flow rate of $c a .50 \mathrm{~mL} \mathrm{~min}{ }^{-1}$.

In the case of the LDH particles, the average hydrodynamic particle size and the size distribution were measured by dynamic 
light scattering (DLS) on a Zetasizer instrument (Malvern Instruments), after the particles were dispersed in ethanol via ultrasonication for $30 \mathrm{~min}$. $\mathrm{C}, \mathrm{H}$, and $\mathrm{N}$ contents were determined using the combustion method on a Flash 2000 Elemental Analyser (Thermo Scientific).

\subsection{In vitro drug release studies}

Drug release from the drug-LDH intercalates was performed by dispersing $10 \mathrm{mg}$ of the particles into $150 \mathrm{~mL}$ of phosphate buffered saline (PBS) at $\mathrm{pH}=7.4$ and stirring at $37{ }^{\circ} \mathrm{C}$. At predetermined time points, $3 \mathrm{~mL}$ aliquots were taken out and replaced with fresh pre-heated phosphate buffer to maintain a constant volume. The samples were filtered through a $0.22 \mu \mathrm{m}$ filter, after which the drug concentrations were measured using a UV-vis spectrophotometer (7315 spectrometer, Jenway) at $222 \mathrm{~nm}$ for ibuprofen and $204 \mathrm{~nm}$ for ketoprofen.

For the fibers, $200 \mathrm{mg}$ of each fiber mat was placed into $150 \mathrm{~mL}$ of PBS at $37{ }^{\circ} \mathrm{C}$ under magnetic stirring. At selected time intervals, $3 \mathrm{~mL}$ of the solution was withdrawn for analysis, and $3 \mathrm{~mL}$ of fresh pre-heated phosphate buffer added to maintain a constant volume. Drug concentrations were quantified using UV spectroscopy following identical procedures to those for the LDH systems.

\section{Results and discussion}

\subsection{Synthesis of LDH-drug nanoparticles}

XRD analysis was first used to verify the intercalation of drug ions into the $\mathrm{LDH}$ particles. The XRD patterns are shown in Fig. 2(a). Both drug-LDH samples have well-developed lamellar structures, characterized by a series of basal diffractions at low $2 \theta$ angles and weaker non-basal diffractions at higher angles.

The interlayer spacing $\left(d_{003}\right)$ values of $\mathrm{Mg} / \mathrm{Al} \mathrm{LDH}$ intercalated with inorganic anions (e.g. nitrate) is reported to be $\sim 0.88 \mathrm{~nm}^{42}$ The distance between adjacent layers is larger with ibuprofen or ketoprofen anions in the interlayer space, at 2.19 and $2.15 \mathrm{~nm}$ respectively. This demonstrates that the drug ions were intercalated into the LDH particles, and these values are in good agreement with previous reports. ${ }^{27,43}$ The expanded interlayer separation is due to the molecular size and geometrical arrangement of the anions in the interlayer space. ${ }^{44}$ As the layer thickness of an $\mathrm{LDH}$ is $0.48 \mathrm{~nm},{ }^{45}$ the gallery heights (defined as the interlayer spacing - the layer thickness) of the ibu-LDH and ket-LDH hybrids are $c a .1 .71$ and $1.67 \mathrm{~nm}$, respectively. This suggests that the ibu and ket ions are arranged as a slightly tilted bilayer with their long axes perpendicular to the metal hydroxide layer. ${ }^{46}$ Such an arrangement permits the carboxylate groups to interact with the layer surfaces.

IR spectra are given in Fig. 2(b and c). Fig. 2(b) depicts the FT-IR spectra of ibuprofen and the ibu-LDH nanohybrid. The characteristic bands at 2962, 2919, and $2867 \mathrm{~cm}^{-1}$ for pure ibuprofen are attributed to the alkyl stretching vibrations. The peaks at 1547 and $1402 \mathrm{~cm}^{-1}$ are due to asymmetric and symmetric $\mathrm{RCOOH}$ stretches, respectively. These have been observed at around 1550 and $1400 \mathrm{~cm}^{-1}$ in previous studies, ${ }^{27,47,48}$ and shift to 1541 and $1399 \mathrm{~cm}^{-1}$ in the ibu-LDH nanohybrids. This
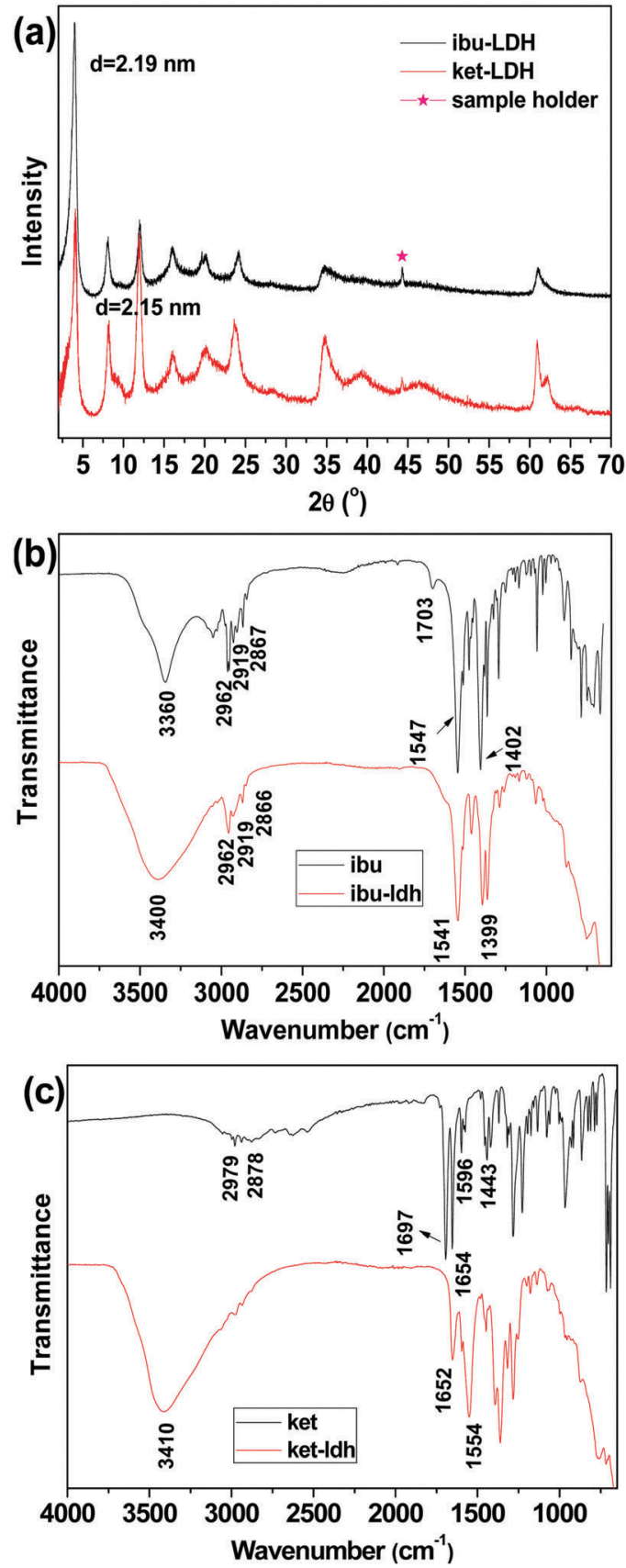

Fig. 2 (a) XRD patterns of ibuprofen and ketoprofen intercalated LDH, with FT-IR spectra of (b) pure ibuprofen and ibu-LDH, and (c) pure ketoprofen and ket-LDH.

is expected to arise as a result of hydrogen bonding and electrostatic interactions between the $\mathrm{RCOO}^{-}$groups and the LDH layers in the intercalate. ${ }^{46}$ The weak bands at 1511 and $1466 \mathrm{~cm}^{-1}$ in the ibuprofen and ibu-LDH spectra are assigned to aromatic ring vibrations.

Pure ketoprofen (Fig. 2(c)) shows characteristic absorption peaks at 3054 and $2979-2878 \mathrm{~cm}^{-1}$, denoting stretching vibrations of aromatic and methyl $\mathrm{C}-\mathrm{H}$ groups. The bands at 1697 and $1654 \mathrm{~cm}^{-1}$ are due to $\mathrm{C}=\mathrm{O}$ stretching of the carboxylic acid and ketone groups respectively. ${ }^{49}$ Peaks at 1596 and $1443 \mathrm{~cm}^{-1}$ 

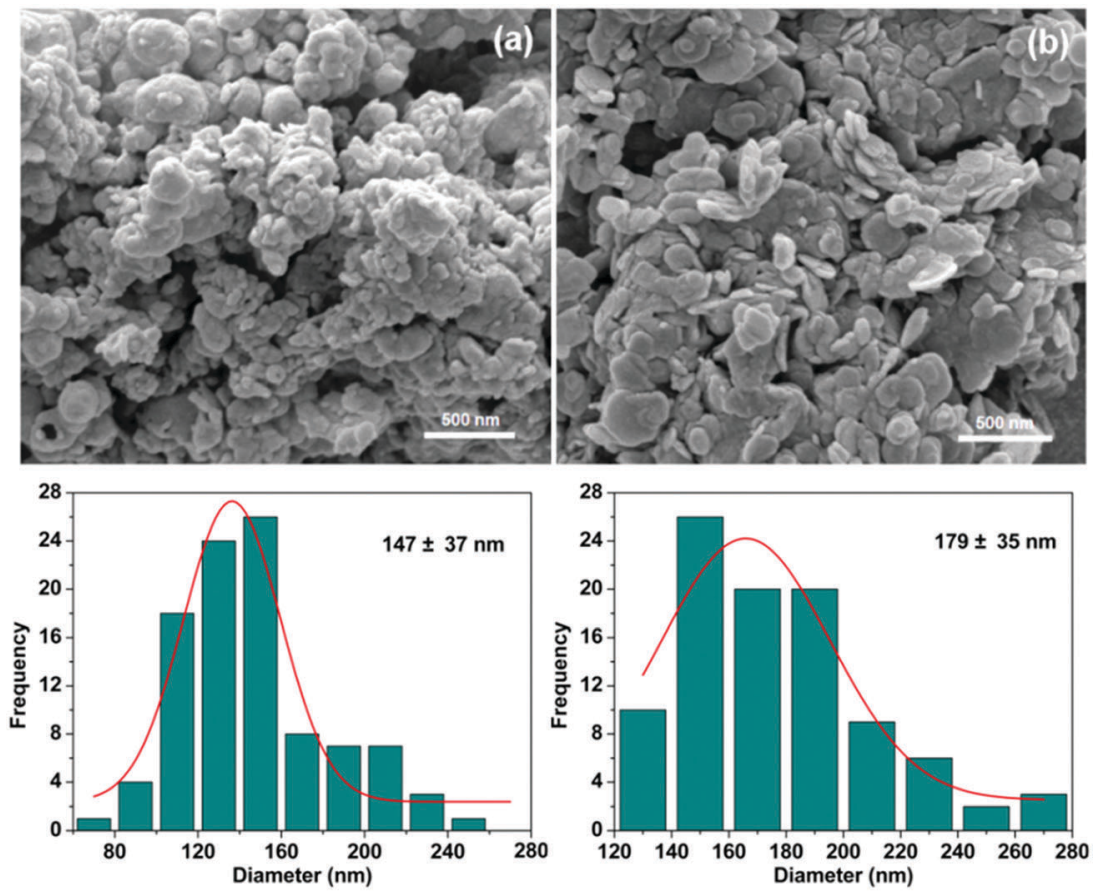

Fig. 3 SEM images of (a) ibu-LDH and (b) ket-LDH, with particle size distributions.

can be attributed to $\mathrm{C}=\mathrm{C}$ stretching of the aromatic ring. As was observed for ibuprofen, the carboxylate stretches shift in position after intercalation, indicating the formation of interactions between the LDH layer and the guest anions.

The IR spectra of both ibu-LDH and ket-LDH also show a very broad band centered at approximately $3400 \mathrm{~cm}^{-1}$, which corresponds to $v(\mathrm{OH})$ stretching vibrations of the hydroxyl groups of the host layers. The observations from IR spectroscopy thus concur with the XRD data, demonstrating successful intercalation of ibu and ket into the LDH gallery. Elemental analysis measurements (Table S1, ESI $\dagger$ ) reveal that the average loading of ibuprofen and ketoprofen in the intercalates was $42.01 \pm 2.55 \%$ and $45.89 \pm 0.76 \% \mathrm{w} / \mathrm{w}$, respectively.

The morphologies of the LDH particles were characterized using SEM analysis (Fig. 3). Both ibu-LDH and ket-LDH exist as aggregated plate-like nanoparticles. The lateral diameters of the particles are $147 \pm 37 \mathrm{~nm}$ and $179 \pm 35 \mathrm{~nm}$, respectively. The hydrodynamic diameters measured by dynamic light scattering (DLS) are rather larger than those determined from the SEM images, with DLS giving sizes of $296 \pm 3 \mathrm{~nm}$ and $315 \pm 13 \mathrm{~nm}$ for the ibu- and ket-LDH systems respectively. This is consistent with previous work, for instance that reported by Xu $e t ~ a l .{ }^{50}$ The larger particle size from DLS can partially be attributed to the particles being hydrated, but the large difference between the SEM and DLS results also suggests that the LDH-drug nanoparticles were not completely dispersed in the DLS experiment, and some aggregates were present.

\subsection{Electrospun fibers}

XRD patterns, given in Fig. 4, provide information on the physical form of the drug and drug-LDH nanohybrids in the polymer matrix. Pure PCL has a semi-crystalline structure with two main reflections at $21.3^{\circ}$ and $23.6^{\circ}$. When fibers were prepared with 2 and $5 \% \mathrm{w} / \mathrm{w}$ ibu (Fig. 4(a)), only the typical peaks of PCL can be seen in the patterns. This shows that the drug is amorphously dispersed in the polymer matrix after electrospinning, most likely as a solid solution, and is consistent with numerous previous reports of drug loaded electrospun fibers. ${ }^{11}$ For the PCL/ibu-LDH fibers, a reflection at $2 \theta=4^{\circ}$ is visible in Fig. 4(a), together with a weak peak at $8^{\circ}$. These correspond to the (003) and (006) reflections of ibu-LDH, indicating that the dispersed LDH retains an ordered structure after electrospinning. They are also more intense with the $10 \% \mathrm{w} / \mathrm{w}$ loading than with $5 \%$, as would be intuitively expected. The overall drug loading in the fibers is calculated to be $4.20 \% \mathrm{w} / \mathrm{w}$ for the PCL $/ 10 \%$ ibu-LDH fibers, and $2.10 \%$ for the PCL $/ 5 \%$ ibu-LDH analogue.

The results with the ketoprofen systems are analogous to those with ibuprofen. The characteristic reflections of ketoprofen were completely absent in the PCL/ket nanofibers (Fig. 4(b)), even at $5 \% \mathrm{w} / \mathrm{w}$ loading, again confirming an amorphous drug distribution. Typical reflections of the ket-LDH hybrid can be seen in the PCL/ket-LDH fibers (Fig. 4(b)), with these being more distinctive at higher loading. The final drug loadings are 4.59 and $2.29 \% \mathrm{w} / \mathrm{w}$ for the PCL $/ 10 \%$ ket-LDH and PCL $/ 5 \%$ ket-LDH materials respectively.

SEM micrographs of the electrospun PCL-drug and PCL/ LDH-drug composites are shown in Fig. 5. All the drug-loaded and LDH/drug-loaded fibers are largely smooth and cylindrical in morphology, but the LDH-free fibers show evidence of solvent inclusion in the form of merged fibers. The average diameter of the fibers ranges from $400 \mathrm{~nm}$ to $1000 \mathrm{~nm}$. There is 

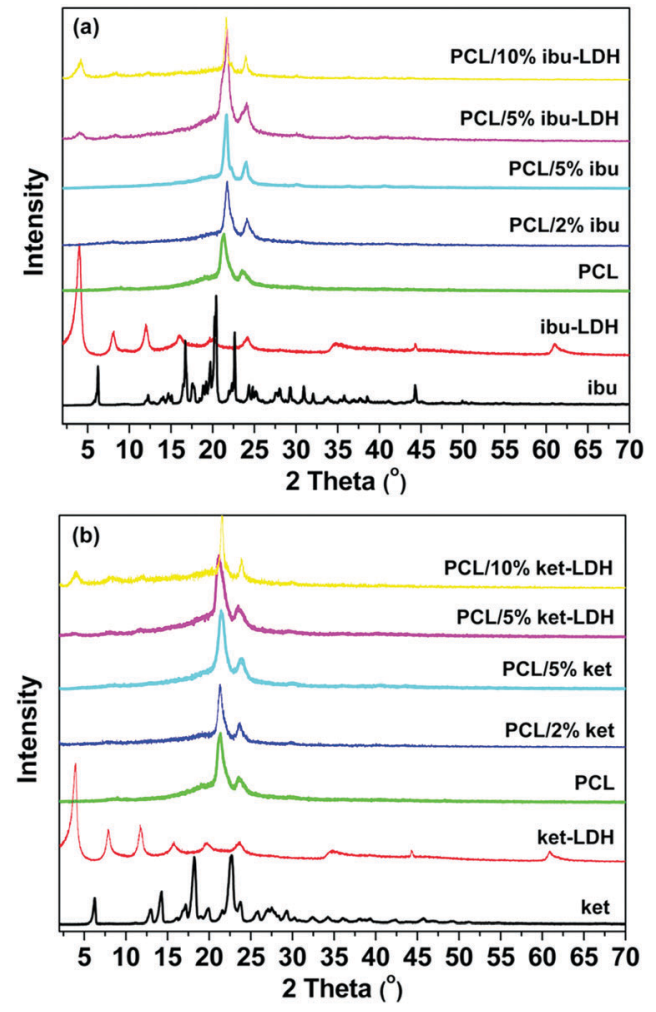

Fig. 4 XRD patterns of the various (a) ibuprofen and (b) ketoprofen containing nanocomposites.

some influence of the drug or LDH-drug loading on the fiber diameters: in most (but not all) cases, the diameter increases with increasing drug or LDH-drug loading. The ketoprofen systems have consistently larger and more homogeneous diameters than their ibuprofen analogues. In general, the LDH-containing fibers have more homogeneous fiber diameters than the analogous LDH-free materials. The reasons for these variations are not entirely clear: the mechanism of electrospinning is extremely complicated, and many factors influence the fiber diameters due to a complex interplay between fluid dynamics, electrodynamics, and rheology. ${ }^{51,52}$

The drug-LDH distribution in the PCL composites was explored using SEM-EDX analyses. Elemental mapping results for $\mathrm{Mg}$ and $\mathrm{Al}$ are given in Fig. $6 . \mathrm{Mg}$ and $\mathrm{Al}$ are distributed evenly throughout the fibers, indicating that the drug loaded LDH nanoparticles were well dispersed as a result of the AMOST process. This renders the surfaces of the LDH particles somewhat hydrophobic, and thus they can efficiently mix with the PCL carrier matrix, rather than aggregating on the surface. In addition, the EDX also showed that the ratio of $\mathrm{Mg} / \mathrm{Al}$ is around 2 .

DSC analyses were performed to evaluate the crystallinity of the fiber systems, and the results are depicted in Fig. 7. The endothermic peak for pure PCL at $63.4{ }^{\circ} \mathrm{C}$ corresponds to its melting point, which is reported to lie the range from 59 to $64{ }^{\circ} \mathrm{C} .{ }^{53,54}$ The DSC curves of ibuprofen and ketoprofen showed sharp endothermic peaks at $77.4{ }^{\circ} \mathrm{C}$ and $94.3{ }^{\circ} \mathrm{C}$ respectively, corresponding to their melting points and confirming the two raw materials to be crystalline. These values agree well with the literature. ${ }^{46,49,55,56}$ The DSC curves of physical mixtures of PCL
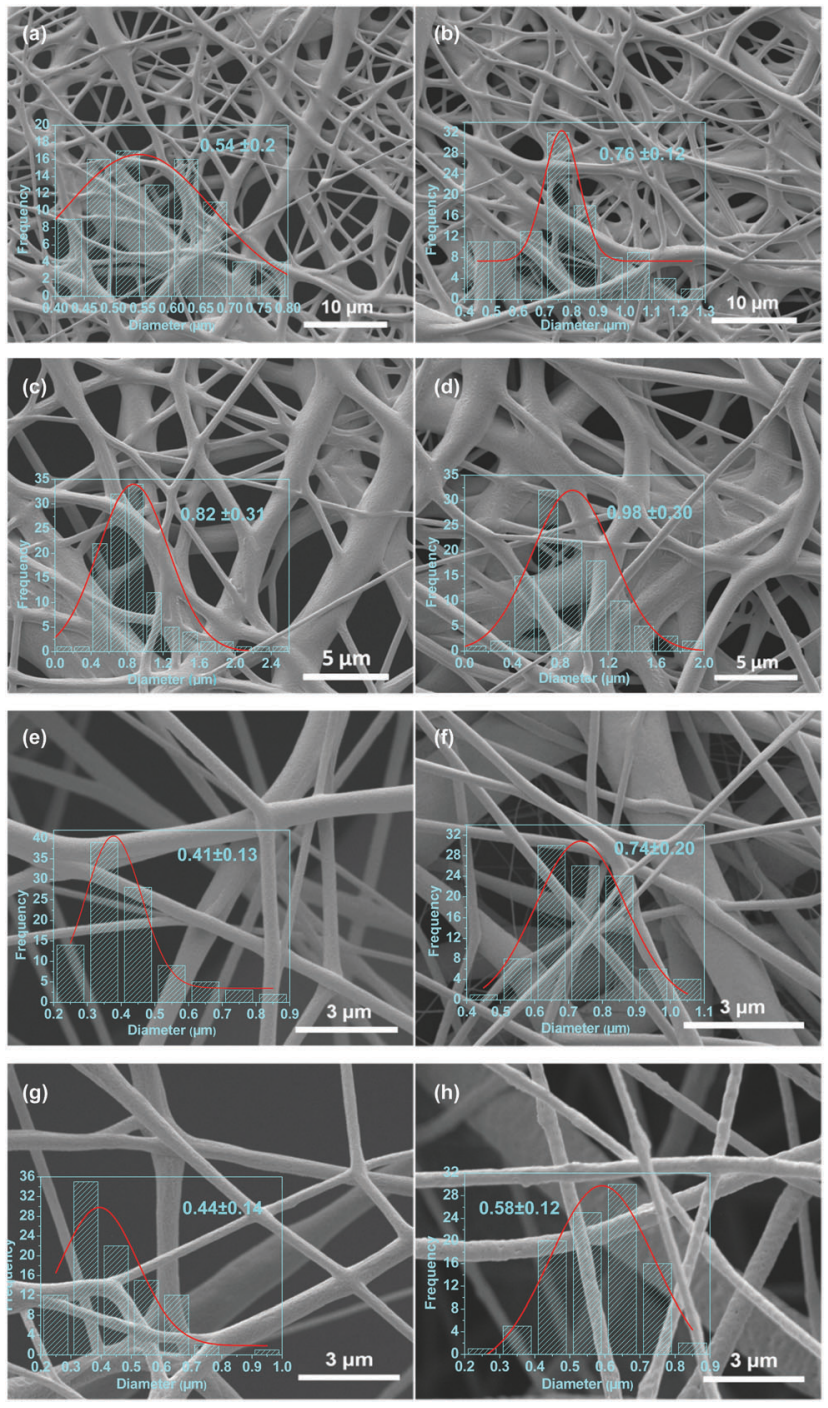

Fig. 5 SEM images of (a) PCL/2\% ibu, (b) PCL/2\% ket, (c) PCL/5\% ibu, (d) $\mathrm{PCL} / 5 \%$ ket, (e) $\mathrm{PCL} / 5 \%$ ibu-LDH, (f) $\mathrm{PCL} / 5 \%$ ket-LDH, (g) PCL/10\% ibu-LDH, and (h) PCL/10\% ket-LDH fibers, with the corresponding diameter distributions.

and either drug show two endothermic peaks, corresponding to the melting points of the two components in the mixture. For the PCL + ibu physical mixture, the endothermic peaks are at 60.8 and $77.0{ }^{\circ} \mathrm{C}$ (Fig. 7(a)), whereas the PCL + ket mixture showed melts at 61.5 and $95.7^{\circ} \mathrm{C}$ (Fig. 7(b)). Thus, as would be expected, simply making a physical mixture does not change the physical form.

In contrast, the DSC curves of the PCL based nanofibers showed only a single peak in the temperature range from 53 to $61{ }^{\circ} \mathrm{C}$, which arises due to melting of the PCL. The peak is slightly shifted to lower temperatures compared to pure PCL, suggesting a reduction in the polymer's crystallinity and the size of its crystals. The degree of crystallinity $\left(X_{\mathrm{c}}\right)$ can be calculated from the melting peak areas using the following equation: ${ }^{57}$

$$
X_{\mathrm{c}}=\frac{\Delta H_{\mathrm{m}}}{\Delta H_{\mathrm{m}}^{\circ} f}
$$



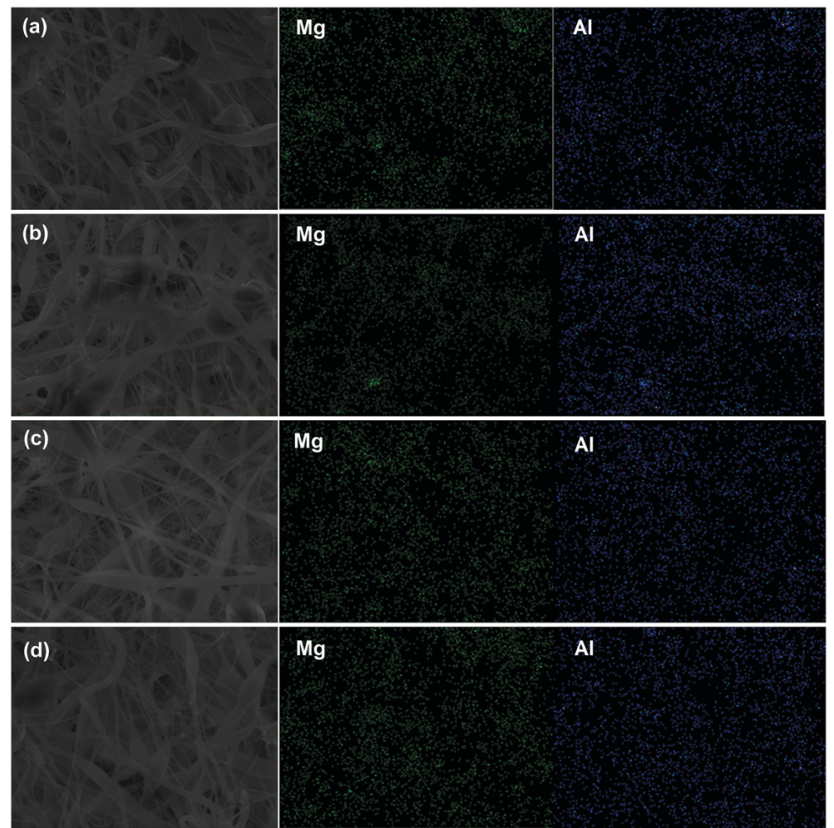

Fig. 6 SEM images, Mg, and Al elemental mapping of the PCL/drug-LDH fibers. (a) $\mathrm{PCL} / 5 \%$ ibu-LDH, (b) $\mathrm{PCL} / 5 \%$ ket-LDH, (c) $\mathrm{PCL} / 10 \%$ ibu-LDH, and (d) $\mathrm{PCL} / 10 \%$ ket-LDH.
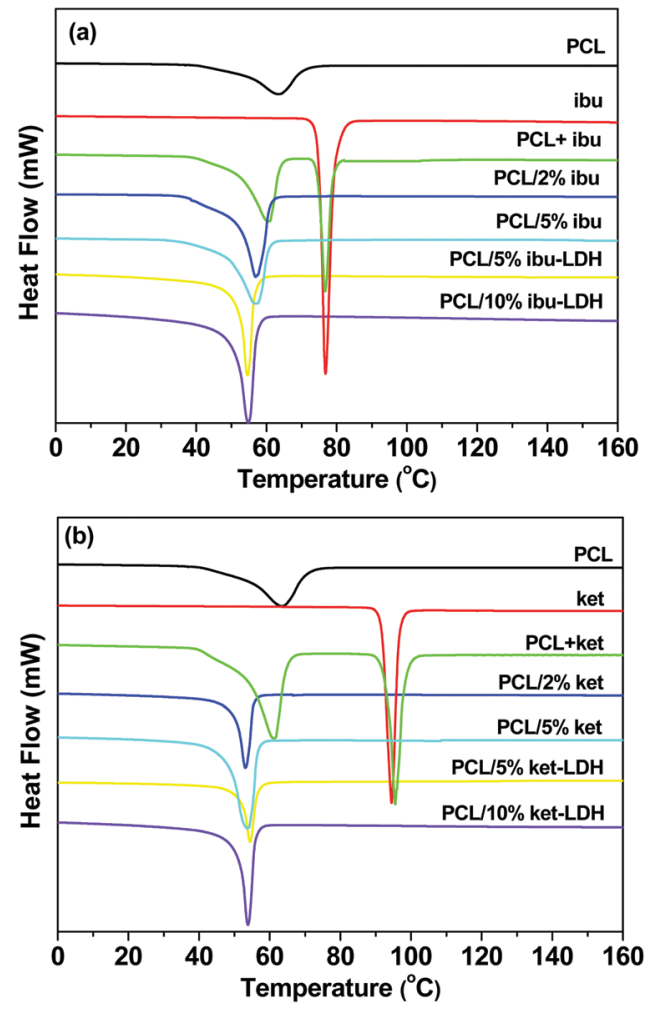

Fig. 7 DSC curves (exo up) of the (a) ibuprofen and (b) ketoprofen based nanocomposites. $\mathrm{PCL}+$ ibu and $\mathrm{PCL}+$ ket denote physical mixtures of the drug and polymer with a mass ratio $5: 1$.

where $\Delta H_{\mathrm{m}}^{\circ}$ is the heat of fusion for a theoretically $100 \%$ crystalline PCL sample (taken to be $146 \mathrm{~J} \mathrm{~g}^{-1}$ ), ${ }^{2} \Delta H_{\mathrm{m}}$ is the melting enthalpy of the sample of interest, and $f$ is the weight
Table 1 Calorimetric data for the various PCL composites

\begin{tabular}{llll}
\hline Samples & $T_{\mathrm{m}}\left({ }^{\circ} \mathrm{C}\right)$ & $\Delta H_{\mathrm{m}}\left(\mathrm{J} \mathrm{g}^{-1}\right)$ & $X_{\mathrm{c}}(\%)$ \\
\hline PCL & 63.43 & 79.35 & 54.35 \\
PCL/2\% ibu & 56.91 & 68.17 & 47.64 \\
PCL/5\% ibu & 56.84 & 56.37 & 40.64 \\
PCL/5\% ibu-LDH & 55.19 & 47.99 & 34.60 \\
PCL/10\% ibu-LDH & 54.82 & 47.61 & 36.23 \\
PCL/2\% ket & 53.08 & 54.00 & 37.74 \\
PCL/5\% ket & 53.71 & 52.86 & 38.11 \\
PCL/5\% ket-LDH & 54.41 & 48.49 & 34.96 \\
PCL/10\% ket-LDH & 53.82 & 46.92 & 35.71
\end{tabular}

fraction of polymer in the system. The results of these calculations are listed in Table 1. After incorporation of the drugs or LDH-drug nanohybrids in the PCL matrix, both $\Delta H_{\mathrm{m}}$ and $X_{\mathrm{c}}$ decreased, indicating that there are some interactions between PCL and the drug or drug-LDH particles.
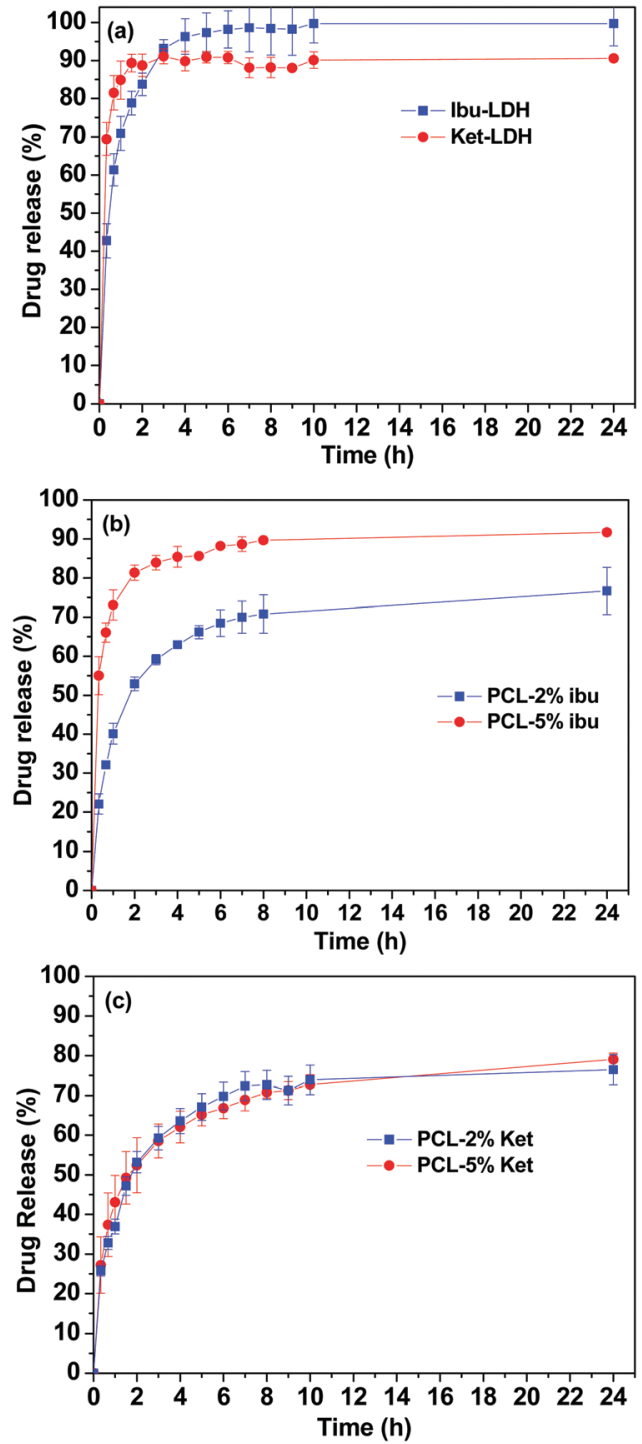

Fig. 8 Drug release profiles of (a) the drug-LDH nanoparticles, (b) PCL/ ibu fibers, and (c) PCL/ket fibers. The data points represent the mean \pm S.D. of three independent experiments. 
The melting endotherms of ibuprofen and ketoprofen were both absent from the PCL/drug fibers. This concurs with the XRD data and confirms the PCL/ket and PLC/ibu fibers to be amorphous solid dispersions. Considering the PCL/LDH-drug fibers, again no endotherms are visible other than the melt of PCL. This is because the drug ions in the LDH interlayer spaces do not adopt the same arrangement as in the pure drug crystals, and the major degradation events of the LDH lie above the upper temperature of the DSC experiment.

\subsection{In vitro drug release}

Fig. 8 shows the cumulative drug release profiles from the drug loaded LDH nanoparticles and the PCL/drug fibers in PBS ( $\mathrm{pH}=7.4$ ) at $37{ }^{\circ} \mathrm{C}$. Significant bursts of release $(>80 \%$ in the first $2 \mathrm{~h}$ ) were seen with both the LDH-drug materials (Fig. 8(a)). Ibu-LDH initially releases more slowly but eventually reaches a higher release percentage than ket-LDH. This can perhaps be explained by the fact that ibuprofen is a smaller ion with greater charge density than ketoprofen, and thus should have stronger interactions with the LDH layers. This will lead to a higher energy barrier to release, causing ibuprofen to release more slowly. At the same time, however, ibuprofen is more water soluble than ketoprofen, and so eventually it is thermodynamically favorable for a larger percentage to release. No further release is observed after $4 \mathrm{~h}$ for both $\mathrm{LDH}-$ drug systems.

Both drugs are released more slowly from the PCL/drug fibers than from the LDH interlayers (Fig. 8(b) and (c)). Over $60 \%$ of the incorporated drug is released within the first $4 \mathrm{~h}$ in all cases. With the PCL/ibu systems, an increase in the drug loading causes an acceleration in the release rate and extent: the PCL $/ 5 \%$ ibu fibers free almost $85 \%$ of their drug content in $4 \mathrm{~h}$. This can be explained because the higher loading will lead to more pores being formed in the fibers as drug molecules escape into solution, making it easier for ibuprofen molecules near the center of the fibers to diffuse to the release milieu. In contrast, the two ketoprofen loadings show essentially identical release profiles. This is likely to be a result of both stronger drug-polymer intermolecular interactions in the case of ketoprofen (PCL is hydrophobic, and ketoprofen more hydrophobic than ibuprofen), and its lower solubility.

The drug release profiles from the PCL/LDH-drug composites are included in Fig. 9. Fig. 9(a) and (b) give the drug release profile over $120 \mathrm{~h}$, while Fig. 9(c) and (d) contain an enlargement of the first $24 \mathrm{~h}$. The PCL/LDH-drug composites are able to reduce the initial burst of release profoundly (Table 2). The materials present a fast release in the initial period of the experiment, followed by a very slow release. The first stage is due to the presence of some LDH-drug particles at or close to the surface of fibers. Drug release from these depends on the ion exchange reaction time, which is very rapid and is completed in about $2 \mathrm{~h}^{2}$ During this period, approximately 13 and $26 \%$ of the incorporated ibuprofen (or 13 and $10 \%$ of ketoprofen) are released from the systems with 5 and $10 \%$ LDH-drug loadings, respectively.

The second and third stages of release start after two hours, and are due to the drug escaping from the inside of the polymer matrix. Here, the release time depends on a number of factors:
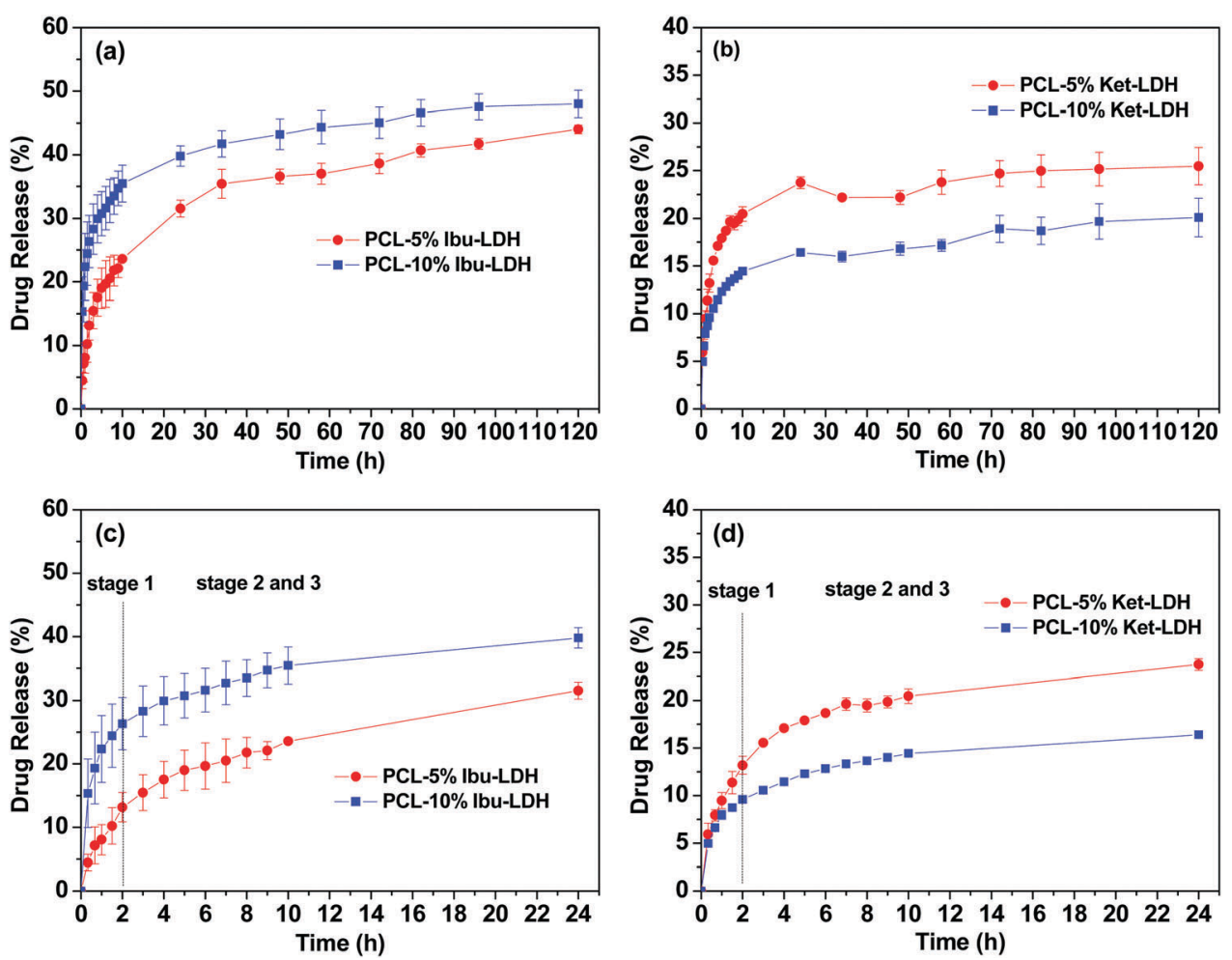

Fig. 9 Drug release profiles for (a) PCL/ibu-LDH over 5 days, (b) PCL/ket-LDH over 5 days, with enlargements of the first $24 \mathrm{~h}$ for (c) PCL/ibu-LDH, and (d) PCL/ket-LDH. The data points represent the mean \pm S.D. of three independent experiments. 
Table 2 A summary of key parameters from in vitro dissolution experiments

\begin{tabular}{lllll}
\hline & \multicolumn{4}{l}{$\%$ drug release after } \\
\cline { 2 - 5 } Sample & $1 \mathrm{~h}$ & $2 \mathrm{~h}$ & $3 \mathrm{~h}$ & $4 \mathrm{~h}$ \\
\hline Ibu-LDH & 71 & 84 & 96 & 98 \\
Ket-LDH & 85 & 88 & 89 & 87 \\
PCL/2\% ibu & 40 & 53 & 63 & 71 \\
PCL/5\% ibu & 73 & 82 & 85 & 89 \\
PCL/2\% ket & 36 & 53 & 64 & 73 \\
PCL/5\% ket & 43 & 52 & 62 & 70 \\
PCL/5\% ibu-LDH & 8 & 13 & 18 & 22 \\
PCL/10\% ibu-LDH & 22 & 26 & 30 & 34 \\
PCL/5\% ket-LDH & 9 & 13 & 17 & 19 \\
PCL/10\% ket-LDH & 8 & 10 & 12 & 14 \\
\end{tabular}

the diffusion behavior of the replacement phosphate ions entering the polymer/LDH matrix, the ion-exchange reaction time, and the diffusion of the drug ions escaping the fiber. This process is very slow and release extending for several days is observed. ${ }^{2}$ The fibers give an almost constant rate of release between $24-120 \mathrm{~h}$, and fitting zero-order kinetics to the data over this period gives $R^{2}$ values $>0.9$ (see Fig. S1, ESI $\dagger$ ). After 5 days, approximately 44 and $48 \%$ of the ibuprofen loading is released for the 5 and $10 \% \mathrm{w} / \mathrm{w}$ fibers, while for ketoprofen the equivalent values are 25 and $20 \%$.

The Ritger-Peppas and Bhaskar equations were also applied to the release data in the first $24 \mathrm{~h}$. The former equation is expressed as: ${ }^{43}$

$$
M_{t} / M_{\infty}=k t^{n}
$$

where $M_{t} / M_{\infty}$ represents the fraction of drug released at time $t$, $k$ is a rate constant and the exponent $n$ gives information on the underlying release mechanisms. The parameters $k$ and $n$ can be calculated by plotting $\log \left(M_{t} / M_{\infty}\right)$ against $\log (t) .{ }^{43,58}$

The Bhaskar model assumes that diffusion through the particle is the rate limiting step to release, making it appropriate for ion-exchange processes. It takes the form: ${ }^{59}$

$$
1-\frac{M_{t}}{M_{\infty}}=\exp \left[-16.33\left(\frac{k}{l^{2}}\right)^{0.65}\right]
$$

where $M_{t} / M_{\infty}$ represents the fraction of drug released at time $t, k$ is the rate constant and $l$ is the particle size in the $a b$-direction, calculated from the $d_{110}$ reflection.

Fig. S2 (ESI $\dagger$ ) depicts the fits obtained with both the RitgerPeppas and Bhaskar equations: while the fits with the former are generally good with $R^{2}>0.96$, the latter are clearly nonlinear, with $R^{2}$ typically below 0.9 . The Peppas exponents are mostly below 0.45 , which for a cylindrical dosage form such as a fiber is consistent with release governed by Fickian diffusion. The complexity of the system makes it difficult to be certain of the drug release mechanism, but the fact that the Peppas model gives good fits while the Bhaskar equation does not might indicate that the diffusion of drug through the PCL fibers is the rate limiting step to release (rather than ion-exchange in the LDH particles).

The use of the AMOST approach leads to significant improvements over previous work in this area: in the previous work by Miao et al., ${ }^{11}$ it was found that the PCL/LDH nanofibers could slow the release of ibuprofen, but very rapid release was still observed in the first 1-2 h, and a plateau reached after $2 \mathrm{~h}$. In contrast, our systems give a constant and sustained rate of release over at least 5 days, giving them great potential as implantable drug delivery systems.

\section{Conclusions}

Sustained drug release materials based on PCL fibers containing drug-intercalated LDH nanoparticles have been prepared and fully characterized. The fibers are found to be smooth and cylindrical, with the LDH-containing systems having more homogenous fibers than those without the inorganic filler. These systems are able to profoundly extend the drug release period over both PCL/drug fibers and LDH-drug particles alone. The use of ternary PCL/LDH-drug formulations also reduces the initial burst of release seen with the binary systems. More than $80 \%$ of the ibuprofen and ketoprofen loadings were released from the LDH-drug particles within $2 \mathrm{~h}$, while release from the PCL/drug fibers reached at least $60 \%$ after $4 \mathrm{~h}$ and plateaus after $10 \mathrm{~h}$. In contrast, the PCL/LDH-drug materials free only $10-26 \%$ of their drug loading after $2 \mathrm{~h}$ and extend release over more than 5 days. The PCL/ibu-LDH materials in particular give an almost constant rate of release from 10-120 h, and thus may have great potential in developing implantable drug delivery systems.

\section{Conflicts of interest}

There are no conflicts to declare.

\section{Acknowledgements}

The authors would like to thank Mr David McCarthy and Mrs Kate Keen for the provision of SEM images, and Dr Eranka Illangakoon (UCL Mechanical Engineering) and Dr Tom Gregory (UCL Archaeology) for assistance with EDX analyses. We also thank the China Scholarship Council, British Council, and Royal Society of Chemistry for funding YG's work at UCL.

\section{References}

1 T. K. Dash and V. B. Konkimalla, Mol. Pharmaceutics, 2012, 9, 2365-2379.

2 L. Tammaro, G. Russo and V. Vittoria, J. Nanomater., 2009, 2009, 33-40.

3 S. R. Baker, S. Banerjee, K. Bonin and M. Guthold, Mater. Sci. Eng., C, 2016, 59, 203-212.

4 T. Potrč, S. Baumgartner, R. Roškar, O. Planinšek, Z. Lavrič, J. Kristl and P. Kocbek, Eur. J. Pharm. Sci., 2015, 75, 101-113.

5 E. G. Bajsic, B. Mijovic, N. V. Penava, T. H. Grguric, M. Slouf and E. Zdraveva, J. Appl. Polym. Sci., 2016, 133, 43539.

6 D. G. Yu, G. R. Williams, X. Wang, X. K. Liu, H. L. Li and S. W. A. Bligh, RSC Adv., 2013, 3, 4652-4658.

7 M. Jin, D. G. Yu, C. F. G. C. Geraldes, G. R. Williams and S. W. A. Bligh, Mol. Pharmaceutics, 2016, 13, 2457-2465. 
8 A. Baskakova, S. Awwad, J. Q. Jiménez, H. Gill, O. Novikov, P. Y. Khaw, S. Brocchini, E. Zhilyakova and G. R. Williams, Int. J. Pharm., 2016, 502, 208-218.

9 M. Mirjalili and S. Zohoori, J. Nanostruct. Chem., 2016, 6, 207-213.

10 J. Cheng, Y. Yesl Jun, J. H. Qin and S.-H. Lee, Biomaterials, 2017, 114, 121-143.

11 Y. E. Miao, H. Zhu, D. Chen, R. Y. Wang, W. W. Tjiu and T. X. Liu, Mater. Chem. Phys., 2012, 134, 623-630.

12 A. I. Khan, L. X. Lei, A. J. Norquist and D. O'Hare, Chem. Commun., 2001, 2342-2343.

13 C. Wang, K. W. Yan, Y. D. Lin and P. C. H. Hsieh, Macromolecules, 2010, 43, 6389-6397.

14 Y. Z. Zhang, X. Wang, Y. Feng, J. Li, C. T. Lim and S. Ramakrishna, Biomacromolecules, 2006, 7, 1049-1057.

15 W. J. Yang, N. Y. He and Z. Y. Li, J. Nanosci. Nanotechnol., 2016, 16, 9404-9412.

16 G. Z. Yang, J. J. Li, D. G. Yu, M. F. He, J. H. Yang and G. R. Williams, Acta Biomater., 2017, 53, 233-241.

17 D. Pedersbæk, M. T. Frantzen and P. Fojan, J. Self-Assem. Mol. Electron., 2017, 5, 17-30.

18 M. Maleki, M. Latifi, M. Amani-Tehran and S. Mathur, Polym. Eng. Sci., 2013, 53, 1770-1779.

19 D. G. Yu, X. Y. Li, X. Wang, J. H. Yang, S. W. Bligh and G. R. Williams, ACS Appl. Mater. Interfaces, 2015, 7, 18891-18897.

20 B. T. Song, C. T. Wu and J. Chang, Regener. Biomater., 2015, 2, 229-237.

21 Y. Z. Wang, B. C. Wang, W. L. Qiao and T. Y. Yin, J. Pharm. Sci., 2010, 99, 4805-4811.

22 G. Polacco, M. G. Cascone, L. Lazzeri, S. Ferrara and P. Giusti, Polym. Int., 2002, 51, 1464-1472.

23 A. I. Khan and D. O'Hare, J. Mater. Chem., 2002, 12, 3191-3198.

24 Q. Wang and D. O'Hare, Chem. Rev., 2012, 112, 4124-4155.

25 G. Simoneau, Eur. J. Drug Metab. Pharmacokinet., 1996, 21, 351-357.

26 K. Ladewig, Z. P. Xu and G. Q. Lu, Expert Opin. Drug Delivery, 2009, 6, 907-922.

27 V. Ambrogi, G. Fardella, G. Grandolini and L. Perioli, Int. J. Pharm., 2001, 220, 23-32.

28 M. Wei, S. Shi, J. Wang, Y. Li and X. Duan, J. Solid State Chem., 2004, 177, 2534-2541.

29 M. S. Birajdar, S. D. Wanjale and S. P. Lonkar, J. Appl. Polym. Sci., 2013, 130, 4508-4515.

30 Q. Qin, Y. Liu, S. C. Chen, F. Y. Zhai, X. K. Jing and Y. Z. Wang, J. Appl. Polym. Sci., 2012, 126, 1556-1563.

31 V. Romeo, G. Gorrasi and V. Vittoria, Biomacromolecules, 2007, 8, 3147-3152.

32 W. Y. Lv, Q. Q. Mei, M. Du, J. L. Xiao, W. J. Ye and Q. Zheng, J. Phys. Chem. C, 2016, 120, 14435-14443.

33 S. S. Shafieia, M. Shavandia, G. Ahangarib and F. Shokrolahic, Appl. Clay Sci., 2016, 127-128, 52-63.

34 E. Valarezo, L. Tammaro, S. Silvia González, O. Malagón and V. Vittoria, Appl. Clay Sci., 2013, 72, 104-109.

35 C. Y. Yang, T. H. Zhu, J. H. Wang, S. H. Chen and W. Y. Li, RSC Adv., 2015, 5, 69423-69429.
36 Q. Wang and D. O'Hare, Chem. Commun., 2013, 49, 6301-6303.

37 Q. Wang, X. Zhang, J. H. Zhu, Z. H. Guo and D. O'Hare, Chem. Commun., 2012, 48, 7450-7452.

38 Y. S. Gao, Q. Wang, J. Y. Wang, L. Huang, X. R. Yan, X. Zhang, Q. L. He, Z. P. Xing and Z. H. Guo, ACS Appl. Mater. Interfaces, 2014, 6, 5094-5104.

39 Y. S. Gao, Q. Wang, L. Qiu, J. W. Wu, X. R. Yan, A. Umar, J. Guo, X. Zhang, J. Y. Wang and Z. H. Guo, Polym. Compos., 2016, 37, 3449-3459.

40 Y. S. Gao, Y. Zhang, G. R. Williams, D. O'Hare and Q. Wang, Sci. Rep., 2016, 6, 35502.

41 Z. P. Xu, G. Stevenson, C. Q. Lu and G. Q. (Max) Lu, J. Phys. Chem. B, 2006, 110, 16923-16929.

42 Y. S. Gao, J. W. Wu, Z. Zhang, R. Jin, X. Zhang, X. R. Yan, A. Umar, Z. H. Guo and Q. Wang, J. Mater. Chem. A, 2013, 1, 9928-9934.

43 M. Silion, D. Hritcu, I. M. Jaba, B. Tamba, D. Ionescu, O. C. Mungiu and I. M. Popa, J. Mater. Sci.: Mater. Med., 2010, 21, 3009-3018.

44 Q. Wang, Z. Wu, H. H. Tay, L. Chen, Y. Liu, J. Chang, Z. Zhong, J. Luo and A. Borgna, Catal. Today, 2011, 164, 198-203.

45 S. Bégu, A. A. Pouëssel, R. Polexe, E. Leitmanova, D. A. Lerner, J.-M. Devoisselle and D. Tichit, Chem. Mater., 2009, 21, 2679-2687.

46 X. M. Lu, L. M. Meng, H. P. Li, N. Du, R. J. Zhang and W. G. Hou, Mater. Res. Bull., 2013, 48, 1512-1517.

47 C. R. Gordijo, C. A. S. Barbosa, A. M. D. C. Ferreira, V. R. L. Constantino and D. d. O. Silva, J. Pharm. Sci., 2005, 94, 1135-1148.

48 V. H. DeLeona, T. D. Nguyena, M. Narb, N. A. D’Souzab and T. D. Golden, Mater. Chem. Phys., 2012, 132, 409-415.

49 G. Vijayaranga Vittal, R. Deveswaran, S. Bharath, B. V. Basavaraj and V. Madhavan, Int. J. Pharm. Invest., 2012, 2, 150-156.

50 Z. Zi, Gu, A. H. Wu, L. Li and Z. P. Xu, Pharmaceutics, 2014, 6, 235-248.

51 S. Ramakrishna, K. Fujihara, W. E. Teo, T. C. Lim and Z. Ma, An introduction to electrospinning and nanofibers, World Scientific Publishing Co. Pte. Ltd, Singapore, 2005.

52 M. Jin, D. G. Yu, X. Wang, C. F. G. C. Geraldes, G. R. Williams and S. W. A. Bligh, Adv. Healthcare Mater., 2016, 5, 977-985.

53 V. R. Sinha, K. Bansal, R. Kaushik, R. Kumria and A. Trehan, Int. J. Pharm., 2004, 278, 1-23.

54 M. A. Woodruff and D. W. Hutmacher, Prog. Polym. Sci., 2010, 35, 1217-1256.

55 M. Subhabrota, R. Souvik and C. Subhadeep, Daru, J. Pharm. Sci., 2011, 19, 47-56.

56 M. L. Vueba, L. A. E. Batista de Carvalho, F. Veiga, J. J. Sousa and M. E. Pina, Eur. J. Pharm. Biopharm., 2004, 58, 51-59.

57 S. K. Nayak, S. Mohanty and S. K. Samal, Mater. Sci. Eng., A, 2009, 523, 32-38.

58 X. Y. Yang, H. M. Trinh, V. Agrahari, Y. Sheng, D. Pal and A. K. Mitra, AAPS PharmSciTech, 2016, 17, 294-306.

59 R. Bhaskar, S. R. S. Murthy, B. D. Miglani and K. Viswanathan, Int. J. Pharm., 1986, 28, 59-66. 